\title{
CONTROL DEL DOLOR POSTQUIRÚRGICO EN PACIENTES DE UN HOSPITAL DE TERCER NIVEL
}

\author{
Jorge Machado-Alba ${ }^{i^{*}}$, Manuel Machado-Duquez, Viviana Calderón ${ }^{2}$, Alexandra González', \\ FELIPE CARDONA², Richard Ruiz', Julián Montoya²
}

\begin{abstract}
${ }^{1}$ Médico, MSc Farmacoepidemiología, MSc en Farmacología. Grupo de Investigación en Farmacoepidemiología y Farmacovigilancia. Universidad Tecnológica de Pereira- Audifarma S.A. Universidad Autónoma de Barcelona.

${ }^{2}$ Estudiante Medicina. Universidad Tecnológica de Pereira.

*Correspondencia: machado@utp.edu.co
\end{abstract}

Recibido: Diciembre 28 de 2012 Aceptado: Febrero 16 de 2013

\begin{abstract}
Resumen
Introducción: el manejo del dolor debe ser adaptado y optimizado de acuerdo con las condiciones de cada hospital, tipo de cirugía y paciente. Objetivos: evaluar la percepción del dolor de los pacientes intervenidos en el postoperatorio del Hospital Universitario San Jorge de Pereira. Materiales y métodos: Se realizó un estudio de corte, prospectivo en pacientes mayores de 18 años entre 2 de septiembre y 28 de octubre de 2011. La valoración de intensidad del dolor postoperatorio se realizó mediante Escala Visual Analógica a las 24 horas del postquirúrgico. Se consideraron variables sociodemográficas, clínicas (tipo de cirugía, anestesia, riesgo) y farmacológicas (medicamentos empleados, dosis, intervalos dosificación). El análisis se hizo con SPSS 20.0 para Windows. Resultados: Se evaluaron 153 pacientes en postoperatorio, $80(52,3 \%)$ mujeres y $73(47,7 \%)$ hombres, con edad promedio de 47,6 $\pm 20,2$ años. El 38,8\% de los pacientes no tenía controlado el dolor. Las variables de cirugía ortopédica, el empleo de anestesia general por vía intravenosa, y el incumplimiento de los intervalos recomendados de dosificación de los analgésicos, se asociaron de manera estadísticamente significativa con la falta de control. Discusión: El control del dolor fue inadecuado haciendo evidente la necesidad de replantear su manejo ajustado a guías de práctica clínica, formalizando el uso de medicamentos, en dosis e intervalos adecuados que garanticen una analgesia efectiva.
\end{abstract}

Palabras clave: Dimensión del dolor, dolor postoperatorio, analgésicos, analgésicos opioides, guía de práctica clínica, agentes antiinflamatorios no esteroides.

\section{CONTROL OF POSTOPERATIVE PAIN IN PATIENTS IN A TERTIARY HOSPITAL}

\begin{abstract}
Introduction: Pain management should be adapted and optimized according to the conditions of each hospital, type of surgery and patient. Objectives: To evaluate the perception of pain in postoperative patients at Hospital Universitario San Jorge of Pereira. Materials and methods: A descriptive observational study in patients older than 18 years, between September 2nd to October 28, 2011. The assessment of postoperative pain intensity was performed by visual analogue scale 24 hours after surgery. We considered socio-demographic, clinical (type of surgery, anesthesia, risk) and pharmacological variables (drugs used, dosage, dosage intervals). The analysis was done with SPSS 20.0 for Windows. Results: We evaluated 153 patients in the postoperative period, 80 (52.3\%) women and 73 (47.7\%) males, mean age $47.6 \pm 20.2$ years. $38.8 \%$ of patients did not obtain pain control. The variables of orthopedic surgery, the use of intravenous general anesthesia, and suboptimal dosage was statistically significantly associated with lack of control. Conclusions: Pain control was inadequate thus showing the
\end{abstract}


Machado J., Machado M., Calderón V., González A., Cardona F., Ruiz R., Montoya J.

need to redefine its management by following clinical practice guidelines, through the use of analgesics in doses and appropriate intervals.

Key words: Pain measurement, visual analogue pain scale, analgesics, opioid, non-steroidal antiinflammatory agents, clinical practice guideline, nonsteroidal anti-inflammatory agents.

\title{
CONTROLE DA DOR PÓS-CIRÚRGICA EM PACIENTES DE UM HOSPITAL DE TERCEIRO NÍVEL
}

\begin{abstract}
Resumo
Introdução: o manejo da dor deve ser adaptado e otimizado de acordo com as condições de cada hospital, tipo de cirurgia e paciente. Objetivos: avaliar a percepção da dor dos pacientes intervindos no pós operatório do Hospital Universitário San Jorge de Pereira. Materiais e métodos: Realizou-se um estudo de corte, prospectivo em pacientes maiores de 18 anos entre 2 de setembro e 28 de outubro de 2011. A valoração de intensidade da dor pós-operatória se realizou mediante Escala Visual Analógica às 24 horas do pós-cirúrgico. Consideraram-se variáveis sociodemográficas, clínicas (tipo de cirurgia, anestesia, risco) e farmacológicas (medicamentos empregados, doses, intervalos, dosagem). A análise se fez com SPSS 20.0 para Windows. Resultados: Se avaliaram 153 pacientes em pós-operatório, 80 $(52,3 \%)$ mulheres e 73 (47,7\%) homens, com idade média de 47,6 $\pm 20,2$ anos. O 38,8\% dos pacientes não tinha controlada a dor. As variáveis de cirurgia ortopédica, o emprego de anestesia geral por via intravenosa, e o descumprimento dos intervalos recomendados de dosagem dos analgésicos, se associaram de maneira estatisticamente significativa com a falta de controle. Discussão: $\mathrm{O}$ controle da dor foi inadequado fazendo evidente a necessidade de repensar o seu manejo ajustado a guias de prática clínica, formalizando o uso de medicamentos, em doses e intervalos adequados que garantam uma analgesia efetiva.
\end{abstract}

Palavras chave: Dimensão da dor, dor pós-operatória, analgésicos, analgésicos opiáceos, guia de prática clínica, agentes anti-inflamatórios não esteroides.

El control del dolor en el postoperatorio es un indicador de la calidad de la atención sanitaria que se le brinda a un paciente y las estrategias para su manejo han avanzado de manera significativa en la actualidad $(1,2)$. Sin embargo, algunos reportes muestran que la prevalencia de dolor moderado a severo después de las cirugías se encuentra alrededor del $26,0 \%$ a $33,0 \%$ $y$ de dolor severo entre $8,0 \%$ y $13,0 \%$ (3).

El inadecuado control del dolor se asocia a complicaciones que afectan la función ventilatoria, la circulación local, el tracto gastrointestinal y urinario, pero también se han asociado con insuficiencia cardiaca, infarto y alteraciones metabólicas, neurológicas, psicológicas y al desarrollo de dolor postoperatorio crónico (4-7). La evidencia actual ha mostrado que el adecuado control del dolor agudo postoperatorio facilita la recuperación, lo cual reduce el tiempo de estancia hospitalaria, los costos y además disminuye la morbilidad y mortalidad asociada al cuidado en un centro asistencial $(7,8)$.
El dolor, considerado también un signo vital clave, ha requerido de la utilización de estrategias para valorar su intensidad y evaluar la calidad del tratamiento recibido, en especial mediante el uso de la denominada, Escala Visual Analógica (EVA) horizontal, que utilizando valores que van de 0 a 100 permite hacer una medición subjetiva de la intensidad del dolor que manifiesta cada paciente $(9,10)$. El hallazgo de una puntuación superior a 40 obliga a evaluar el caso e intervenir rápidamente para conseguir el alivio el síntoma, con lo cual se puede lograr una movilización temprana (11-13).

Existe una amplia variedad de medicamentos útiles para el manejo y control del dolor en el postoperatorio, encabezados por los opioides, analgésicos, antiinflamatorios no esteroideos (AINEs), anestésicos locales e incluso dispositivos que regulan su administración. La recomendación más práctica es la asociación de dos analgésicos con mecanismos de acción distintos, con lo cual se puede conseguir una analgesia mayor y además 
se pueden reducir los efectos indeseables asociados a las dosis de algunos de los fármacos (14-15).

Se pretendió determinar la intensidad de dolor percibida por los pacientes intervenidos quirúrgicamente en el Hospital Universitario San Jorge de Pereira a las 24 horas del postoperatorio mediante una EVA y definir las variables que se asociaron con el control del dolor o la falta de dicho control, con el fin de optimizar su manejo.

\section{Materiales y métodos}

Se realizó un estudio de corte, prospectivo en el Hospital Universitario San Jorge de Pereira (HUSJ), en una población de pacientes mayores de 18 años, de cualquier sexo, intervenidos quirúrgicamente, entre las 7:00 am y las 6:00 pm, desde el 2 de septiembre hasta el 28 de octubre del año 2011. Se empleó una EVA en milímetros $(\mathrm{mm})$ para la valoración de la intensidad del dolor postoperatorio con cinco niveles. El 0 y el 100 representan valores absolutos y son categorías independientes, para lo cual se determinaron los siguientes valores de referencia: $0 \mathrm{~mm}$ como ningún dolor, 1-19 $\mathrm{mm}$ dolor muy leve, $20-39 \mathrm{~mm}$ dolor leve, $40-59 \mathrm{~mm}$ dolor medio, 60-79 $\mathrm{mm}$ dolor fuerte, $80-99 \mathrm{~mm}$ dolor muy fuerte y $100 \mathrm{~mm}$ como el peor dolor posible y se definió como dolor no controlado los valores superiores a $40 \mathrm{~mm}(16-18)$.

Se hizo la evaluación a las 24 horas posteriores a la finalización del procedimiento quirúrgico, con la intención de valorar el manejo del dolor por parte del equipo de salud responsable de la atención en la sala donde quedase hospitalizado el paciente. Fueron excluidos aquellos pacientes que no pudiesen realizar el test por déficit neurológico, trastornos motores incapacitantes, retraso mental y enfermedades mentales graves. La información fue obtenida por estudiantes de último año de medicina de la Universidad Tecnológica de Pereira mediante entrevista al paciente. Además, se revisó la historia clínica del paciente y la nota quirúrgica, previa autorización con la firma de un consentimiento informado. Se empleó un instrumento de recolección de datos elaborado por los investigadores que además de la EVA consideró las siguientes variables:

Sociodemográficas y toxicológicas previas: incluyendo edad, sexo, estado civil (solo o acompañado), régimen de salud (subsidiado o contributivo), nivel socioeconómico (bajo, medio, alto), nivel educativo (primaria, secundaria, superior), residencia (urbana o rural), tabaquismo, consumo de alcohol, consumo de sustancias psicoactivas, consumo de AINEs, corticosteroides y antidepresivos.

Clínicas: tipo de cirugía según especialidad quirúrgica (cirugía general, neurocirugía, urológica, plástica, ortopédica, otorrinolaringológica, ginecológica, etc.), complicaciones durante ésta y en el postoperatorio, tipo de anestesia (general inhalada, intravenosa, conductiva, local, etc.), riesgo estimado de la cirugía (alto, moderado y bajo) para lo cual se determinó el bajo riesgo para la cirugía mínimamente invasiva con perdidas sanguíneas inferiores a $200 \mathrm{cc}$; moderado riesgo en procedimientos moderadamente invasivos con intercambio de fluidos, pérdidas potenciales de sangre de hasta $1000 \mathrm{cc}, \mathrm{y} / \mathrm{o}$ mortalidad/morbilidad moderada relacionada con el procedimiento y alto riesgo para los procedimientos altamente invasivos tales como procedimientos radicales o extensos en el abdomen superior, tórax o cráneo y pérdidas potenciales de sangre superiores a $1000 \mathrm{cc}$ con una mortalidad/morbilidad significativa asociada al procedimiento.

Farmacológicas: medicamentos prescritos en el postoperatorio durante las primeras 24 horas, agrupados por clase y su utilización como monoterapia y terapia combinada, la dosis, el intervalo de dosificación de cada uno, las reacciones adversas medicamentosas asociadas y el uso de premedicación analgésica. Se definió el uso de morfina, meperidina, fentanilo como narcóticos fuertes y de tramadol como narcótico moderado.

El protocolo se sometió a la aprobación del Comité de Bioética de la Facultad de Ciencias de la Salud de la Universidad Tecnológica de Pereira en la categoría de "investigación con riesgo inferior al mínimo", según la resolución No. 008430 de 1993 del Ministerio de Salud de Colombia, que establece las normas científicas, técnicas y administrativas para la investigación en salud.

Para el análisis se utilizó el paquete estadístico SPSS versión 20 para Windows (IBM, EE.UU). Se emplearon las pruebas t de student o ANOVA para la comparación de variables cuantitativas y la prueba de $X^{2}$ para comparar las variables categóricas. Se aplicaron modelos de regresión logística usando como variable dependiente el control del dolor y como variables independientes aquellas que fueron significativas en el análisis bivariado. Se determinó un nivel de significancia estadística de una $\mathrm{p}<0,05$. 


\section{Resultados}

Se evaluaron un total de 153 pacientes intervenidos quirúrgicamente entre los cuales $80(52,3 \%)$ fueron mujeres y $73(47,7 \%)$ hombres, con una edad promedio de 47,6 \pm 20,2 años (rango: 18 a 85 años). En la tabla 1 se pueden observar las características sociodemográficas y clínicas de los pacientes evaluados en el estudio que muestra un predominio de régimen subsidiado, nivel socio económico y nivel educativo bajo, del área urbana de los diferentes municipios del departamento de Risaralda, con bajo consumo previo de alcohol, tabaco y sustancias psicoactivas. Los procedimientos según especialidades quirúrgicas más comunes fueron la cirugía general, ginecológica y ortopédica y el método anestésico más utilizado fue general inhalada. Además, la premedicación anestésica fue poco utilizada.

Tabla 1. Características socio-demográficas, médicas y quirúrgicas de 153 pacientes atendidos a las 24 horas del postoperatorio en el Hospital Universitario San Jorge, Pereira, 2011.

\begin{tabular}{|c|c|c|}
\hline $\begin{array}{c}\text { Características } \\
\text { socio-demográficas }\end{array}$ & $n=153$ & $(\%)$ \\
\hline Género (Hombres/Mujeres) & $73 / 80$ & $47,7 / 52,3$ \\
\hline Edad (promedio $\pm D E$, años) & $47,6 \pm 20,2$ & \\
\hline \multicolumn{3}{|l|}{ Estado civil } \\
\hline Soltero/acompañado & $32 / 121$ & $20,9 / 79,1$ \\
\hline \multicolumn{3}{|l|}{ Régimen de salud } \\
\hline Contributivo/Subsidiado/Sin dato & $9 / 143 / 1$ & $5,9 / 93,5 / 0,7$ \\
\hline \multicolumn{3}{|l|}{ Nivel educativo } \\
\hline Primaria/secundaria/superior & $90 / 56 / 7$ & $58,8 / 36,6 / 4,6$ \\
\hline \multicolumn{3}{|l|}{ Residencia } \\
\hline Urbana/rural/ & $111 / 42$ & $72,5 / 27,5$ \\
\hline \multicolumn{3}{|l|}{ Nivel socioeconómico } \\
\hline $1 / 2 / 3 / 4 / 5 /$ sin dato & $95 / 43 / 10 / 1 / 1 / 3$ & $62,1 / 28,1 / 6,5 / 0,7 / 0,7 / 2,0$ \\
\hline \multicolumn{3}{|l|}{ Antecedentes personales } \\
\hline Tabaquismo & 47 & 30,7 \\
\hline Alcohol & 36 & 23,5 \\
\hline Sustancias Psicoactivas & 10 & 6,5 \\
\hline \multicolumn{3}{|l|}{ Antecedentes farmacológicos } \\
\hline AINEs & 31 & 20,3 \\
\hline Glucocorticoides & 6 & 3,9 \\
\hline \multicolumn{3}{|l|}{ Tipo de cirugía } \\
\hline Ortopedia & 61 & 39,9 \\
\hline General & 50 & 32,7 \\
\hline Ginecológica & 20 & 13,1 \\
\hline Urología & 11 & 7,2 \\
\hline Plástica & 4 & 2,6 \\
\hline Neurocirugía & 4 & 2,6 \\
\hline Laparoscópica & 3 & 2,0 \\
\hline \multicolumn{3}{|l|}{ Pre-medicación anestésica } \\
\hline $\mathrm{Si} / \mathrm{no}$ & $7 / 146$ & $4,6 / 95,4$ \\
\hline \multicolumn{3}{|l|}{ Tipo de anestesia } \\
\hline General inhalada & 68 & 44,4 \\
\hline Conductiva & 61 & 39,9 \\
\hline General intravenosa & 15 & 9,8 \\
\hline Regional & 5 & 3,3 \\
\hline Local & 4 & 2,6 \\
\hline
\end{tabular}


La medición del síntoma mediante la EVA a las 24 horas, encontró un nivel de dolor promedio de 29,7 mm y se hallaron 59 (38,6\%) pacientes sin control del dolor y 94 (61,4\%) controlados; además se observó que 25 pacientes $(16,3 \%)$ exigieron analgesia de rescate durante el periodo de hospitalización a consecuencia de la intensidad del dolor. En la figura 1 se puede observar la distribución de los porcentajes de pacientes según el rango de dolor encontrado en la evaluación. En la tabla 2 se muestran los analgésicos utilizados, el número de medicamentos que recibió cada paciente y sus asociaciones; la dipirona fue el analgésico más empleado en monoterapia y en terapia combinada.

Figura 1. Porcentaje de pacientes según intensidad del dolor a las 24 horas del postoperatorio en 153 pacientes del Hospital Universitario San Jorge, Pereira, 2011.

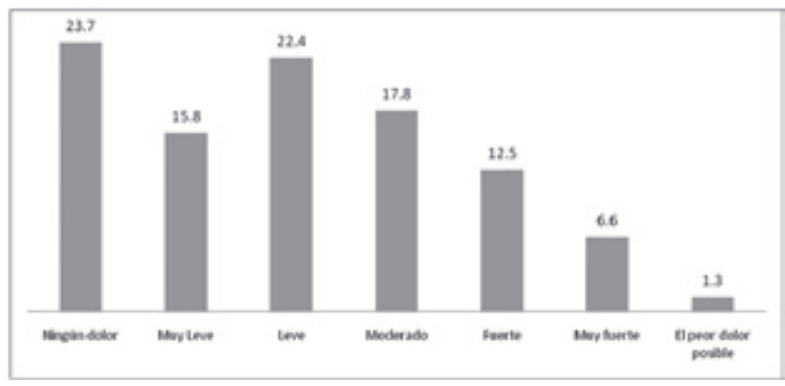

Tabla 2. Medicamentos y esquemas más empleados a las 24 horas del postoperatorio de 153 pacientes atendidos en el Hospital Universitario San Jorge, Pereira, 2011.

\begin{tabular}{|l|c|c|}
\hline Variables farmacológicas & $\mathbf{n}=\mathbf{1 5 3}$ & $\mathbf{\%}$ \\
\hline Número medicamentos por paciente & & \\
\hline Ninguno & 9 & 5,9 \\
\hline 1 & 92 & 60,1 \\
\hline 2 & 47 & 30,9 \\
\hline 3 & 5 & 3,1 \\
\hline Analgésicos empleados & $\mathrm{n}=204$ & \\
\hline Dipirona & 136 & 66,7 \\
\hline Tramadol & 32 & 15,7 \\
\hline Acetaminofén & 20 & 9,8 \\
\hline Diclofenaco & 12 & 5,9 \\
\hline Morfina & 4 & 1,9 \\
\hline Esquemas empleados con mayor frecuencia & & \\
\hline Solo analgésico antipirético & 89 & 58,6 \\
\hline Tramadol + analgésico antipirético & 23 & 15,1 \\
\hline AlNE + analgésico antipirético & 19 & 12,5 \\
\hline Ninguno & 9 & 5,9 \\
\hline Otros & 7 & 4,6 \\
\hline Opioide Fuerte & 3 & 1,9 \\
\hline Opioide Fuerte + analgésico antipirético + AINE & 2 & 1,3 \\
\hline
\end{tabular}

Comparación de pacientes con dolor controlado versus no controlado

En la tabla 3 se presentan los resultados de los análisis bivariados que permiten hacer la comparación de los subgrupos de pacientes con control del dolor versus aquellos sin control. Se encontró que las variables sexo, edad, nivel educativo, antecedentes de consumo de alcohol, psicoactivos, premedicación anestésica, los efectos indeseables por el uso de los analgésicos y las comorbilidades no se asociaron estadísticamente con la falta de control de dolor. Las variables, uso de anestesia general intravenosa y ser intervenido para cirugía ortopédica se asociaron de manera estadísticamente significativa con falta de control del dolor a las 24 horas del postoperatorio, mientras que las variables, uso de anestesia general inhalada, cirugía urológica y cumplir con el intervalo recomendado de dosificación del segundo analgésico se asociaron estadísticamente con el control adecuado del dolor.

El análisis multivariado encontró que la única variable independiente que se asoció de forma estadísticamente significativa con el control del dolor, fue cumplir el intervalo de dosificación recomendado del segundo analgésico empleado (Riesgo Relativo: 0,17; Intervalo de Confianza 95\%: 0,035-0,844; $\mathrm{p}=0,03$ ).

\section{Discusión}

El control del dolor postoperatorio, continúa siendo un serio problema a pesar de la eficacia y seguridad de los medicamentos existentes y su monitoreo riguroso es de suma importancia en cualquier centro especializado, lo cual requiere de la participación de equipos multidisciplinarios $(14,15,19,20)$. La importante prevalencia de dolor postquirúrgico no controlado hallada en esta cohorte, se puede considerar elevada cuando se compara con otros trabajos que muestran que a las 24 horas aproximadamente, el 11,0\% de los pacientes aún no han aliviado este síntoma de manera adecuada. Las posibles explicaciones para la falta de control pueden involucrar el empleo de medicamentos en monoterapia, contrario a lo recomendado por diferentes autores que proponen el manejo con 2 fármacos que tengan diferente mecanismo de acción, o la falta de implementación de nuevas técnicas de control del dolor como las bombas de autoadministración de opiodes, la anestesia epidural torácica o bloqueos paravertebrales $(3,14,15,21-26)$. 
Tabla 3. Análisis bivariado del control del dolor a las 24 horas versus las principales variables socio demográficas y clínicas de 153 pacientes intervenidos quirúrgicamente en el Hospital Universitario San Jorge, Pereira, 2011.

\begin{tabular}{|c|c|c|c|c|c|}
\hline \multirow[b]{2}{*}{ Características } & \multicolumn{2}{|c|}{$\begin{array}{l}\text { Si control de dolor } \\
\text { a las } 24 \text { horas }\end{array}$} & \multicolumn{2}{|c|}{$\begin{array}{l}\text { No control del dolor } \\
\text { a las } 24 \text { horas }\end{array}$} & \multirow[b]{2}{*}{ Valor de $\mathrm{P}^{*}$} \\
\hline & Número & $\%$ & Número & $\%$ & \\
\hline \multicolumn{6}{|l|}{ Género } \\
\hline Masculino & 45 & 61,6 & 28 & 38,4 & 0,96 \\
\hline Femenino & 49 & 61,3 & 31 & 38,8 & \\
\hline \multicolumn{6}{|l|}{ Edades } \\
\hline Adulto Joven (18 - 44) & 39 & 55,7 & 31 & 44,3 & 0,182 \\
\hline Adulto Medio (45 - 64) & 25 & 59,5 & 17 & 40,5 & 0,765 \\
\hline Adulto Mayor (65+) & 28 & 73,7 & 10 & 26,3 & 0,074 \\
\hline \multicolumn{6}{|l|}{ Nivel Educativo } \\
\hline Bajo & 77 & 59,7 & 52 & 40,3 & 0,303 \\
\hline Alto & 17 & 70,8 & 7 & 29,2 & \\
\hline \multicolumn{6}{|l|}{ Especialidad quirúrgica } \\
\hline Urológica & 11 & 100,0 & 0 & 0 & 0,006 \\
\hline Plástica & 3 & 75,0 & 1 & 25,0 & 0,572 \\
\hline General & 32 & 64,0 & 18 & 36,0 & 0,650 \\
\hline Ortopedia & 31 & 50,8 & 30 & 49,2 & 0,028 \\
\hline Gineco-obstétrica & 12 & 60,0 & 8 & 40,0 & 0,887 \\
\hline Laparoscópica & 2 & 66,7 & 1 & 33,3 & 0,851 \\
\hline Neurocirugía & 3 & 75,0 & 1 & 25,0 & 0,572 \\
\hline \multicolumn{6}{|l|}{ Tipo de anestesia } \\
\hline Conductiva & 36 & 59,0 & 25 & 41,0 & 0,616 \\
\hline General Intravenosa & 5 & 33,3 & 10 & 66,7 & 0,019 \\
\hline General Inhalada & 49 & 72,1 & 19 & 27,9 & 0,016 \\
\hline otras & 4 & 44,4 & 5 & 55,6 & \\
\hline \multicolumn{6}{|l|}{$\begin{array}{l}\text { Cumple intervalo dosificación } \\
\text { primer analgésico }\end{array}$} \\
\hline $\mathrm{Si}$ & 74 & 58,7 & 52 & 41,3 & 0,137 \\
\hline No & 20 & 74,1 & 7 & 25,9 & \\
\hline \multicolumn{6}{|l|}{$\begin{array}{l}\text { Cumple intervalo dosificación } \\
\text { segundo analgésico }\end{array}$} \\
\hline $\mathrm{Si}$ & 23 & 62,2 & 14 & 37,8 & 0,025 \\
\hline No & 3 & 25,0 & 9 & 75,0 & \\
\hline \multicolumn{6}{|l|}{ Esquema de analgésicos } \\
\hline Solo analgésico antipirético & 55 & 65,5 & 29 & 34,5 & 0,258 \\
\hline AINE + analgésico antipirético & 10 & 55,6 & 8 & 44,4 & 0,585 \\
\hline Tramadol + analgésico antipirético & 13 & 59,1 & 9 & 40,9 & 0,807 \\
\hline
\end{tabular}

*Basado en el test de chi cuadrado 
La frecuente utilización de dipirona sola y combinada con opiodes, concuerda con lo mostrado en estudios similares colombianos y alemanes, en los cuales el control del dolor no fue adecuado (14,21), sin embargo, discrepa de resultados de estudios españoles y griegos donde los fármacos más prescritos fueron los opiodes solos o asociados a AINES $(9,22,27)$. Es llamativo el porcentaje de pacientes que solicitaron analgesia de rescate, como evidencia de la insuficiente respuesta de un esquema eficaz de control del dolor $(21,22)$, así como es preocupante encontrar nueve pacientes sin ningún tipo de manejo analgésico.

El uso insuficiente e inadecuado de los opioides en este hospital, es reflejado en la prescripción en intervalos subóptimos que no cubren en el horario las necesidades del paciente y demuestran desconocimiento de la farmacocinética que garantice el manejo apropiado de estos fármacos. A lo anterior, se debe sumar el temor a sus reacciones adversas, práctica ya reportada por otros autores con lo que no se consigue el alivio del dolor, pero si mantiene el riesgo de reacciones adversas entre ellas el delirio en ancianos, lo cual puede ser un indicador de la indiferencia que se ha evidenciado por parte del personal sanitario frente al dolor ajeno y el desconocimiento de los derechos del paciente $(14,22$, 25,28-30).

Ortopedia fue la especialidad que realizó procedimientos con más frecuencia en el HUSJ, para la cual se encontró en el presente estudio una asociación significativa con mayor presencia de dolor no controlado. Esta intensidad del dolor es la esperada con base en el tipo de cirugía practicada, ya que involucra factores determinantes de la percepción del dolor como son el extenso daño tisular y el alto gasto metabólico. Aunque hay que tener en cuenta que de todos estos factores, el tipo de intervención, la técnica quirúrgica y el manejo anestésico empleados son los condicionantes de mayor importancia en la intensidad y duración del dolor (31). Las cirugías urológicas se relacionaron con mejor control del dolor, contrario a lo encontrado por otro estudio nacional donde estos procedimientos se asociaron con falta de control (21).

Las principales limitaciones encontradas en este estudio se relacionan con que la EVA solo proporciona una medida unidimensional, examinando solo el componente sensorial y excluye el componente afectivo y cognitivo del paciente, además como se hizo monitoreo de la intensidad del dolor en un momento, no se conoce cuál fue la percepción en el intervalo de tiempo entre la cirugía y las primeras 24 horas y aún después de este intervalo, sumado a la falta de registro de la formulación en algunas historias clínicas. La heterogeneidad misma de los esquemas y medicamentos empleados que es una limitación en la interpretación de los resultados, se convierte en la razón misma de considerar la importancia de adoptar guías de manejo del dolor en el postoperatorio, que faciliten el uso racional de los medicamentos recomendados $(5,8,14)$.

Consideramos que la falta de control del dolor en el postoperatorio, es un problema evidente en el HUSJ, el cual está asociado con algunos procedimientos, en especial la cirugía ortopédica, el empleo de anestesia general por vía intravenosa, y el incumplimiento de los intervalos recomendados de dosificación de los analgésicos, mientras que los procedimientos urológicos y el empleo de anestesia general inhalada se asoció con mayor control del dolor. Se considera necesario replantear la terapia analgésica en el hospital, ajustándose a guías de manejo internacionales o mejor aún, a la creación de guías propias, buscando controlar adecuadamente el dolor agudo en el postoperatorio y darle la importancia requerida para este importante síntoma, mediante el adecuado uso de medicamentos analgésicos, en dosis y pautas de dosificación ajustadas y adecuadas al paciente, y promover incluso la creación de una unidad de dolor agudo, que ya han demostrado la máxima efectividad en el manejo y control integral del paciente y que se encargue de la implementación de las guías, el seguimiento de las mismas y la evaluación de su aplicación y los beneficios y resultados clínicos $(3,15,19,32)$. El uso de guías de práctica clínica para el adecuado manejo analgésico ha demostrado que se puede reducir significativamente el dolor y sus complicaciones (14).

\section{Conflicto de intereses: los autores manifiestan que no existe ningún conflicto de intereses.}

\section{Referencias}

1. International Association for the Study of pain. Seattle: Merskey H, Bogduk N. IASP taxonomy. [Internet]. 1994. [acceso 16 de febrero de 2012]. Disponible en: http://www.iasp-pain.org/ Content/NavigationMenu/GeneralResourceLinks/ PainDefinitions/ default.htm.

2. Carr DB, Goudas LC. Acute pain Review. Lancet. 1999; 353(9169): 2051-8.

3. Dolin SJ, Cashman JN, Bland JM. Effectiveness of acute postoperative pain management: I. Evidence from published data. Br J Anaesth. 2002; 89(3): 409-23 
4. Muñoz-Blanco F, Salmerón J, Santiago J, Marcote C. Complicaciones del dolor postoperatorio. Rev Soc Esp Dolor. 2001; 8: 194-211.

5. Martinez-Vazquez de Castro J, Torres LM. Prevalencia del dolor postoperatorio. Alteraciones Fisiopatologicas y sus repercusiones. Rev Soc Esp Dolor. 2000; 7: 465-76.

6. Finkel DM, Schlegel HR. El dolor postoperatorio. Conceptos básicos y fundamentos para un tratamiento adecuado. Rev Hosp Gen Agudos Dr. J. M. Ramos Mejía. 2003; 8(1): 1-17.

7. Brandsborg B. Pain following hysterectomy: epidemiological and clinical aspects. Dan Med J. 2012; 59(1): B4374

8. Reyes Fierro A, de la Gala García F. Dolor postoperatorio: analgesia multimodal. Patología del Aparato Locomotor. 2004; 2 (3): 176-188.

9. Gkotsi A, Petsas D, Sakalis V, Fotas A, Triantafyllidis A, Vouros I, et al. Pain point system scale (PPSS): a method for postoperative pain estimation in retrospective studies. J Pain Res. 2012; 5: 503-10

10. Mularski RA, White-Chu F, Overbay D, Miller L, Asch SM, Ganzini L. Measuring pain as the 5 th vital sign does not improve quality of pain management. J Gen Intern Med. 2006; 21(6): 607-12.

11. Craine M, Kerns RD. Pain management improvement strategies in the Veteran's Health Administration. APS Bull. [Internet]. 2003. [acceso 16 de febrero de 2012]. Disponible en http:// www.ampainsoc.org/library/bulletin/sep03/article1.htm

12. Veterans Health Administration. Pain as the 5th vital sign toolkit. October 2000, revised edition. Geriatrics and Extended Care Strategic Healthcare Group, National Pain Management Coordinating Committee. [Internet]. 2005. [acceso 16 de febrero de 2012]. Disponible en: http://www.va.gov/oaa/pocketcard/pain.asp.

13. Kehlet H. Multimodal approach to control postoperative pathophysiology and rehabilitation. Br J Anaesth. 1997; 78(5): 606-17.

14. Usichenko TI, Röttenbacher I, Kohlmann T, Jülich A, Lange $\mathrm{J}$, Mustea A, et al. Implementation of the quality management system improves postoperative pain treatment: a prospective pre-/post-interventional questionnaire study. Br J Anaesth. [revista electronica]. 2012 Oct 9.

15. American Society of Anesthesiologists Task Force on Acute Pain Management. Practice guidelines for acute pain management in the perioperative setting: an updated report by the American Society of Anesthesiologists Task Force on Acute Pain Management. Anesthesiology. 2004; 100: 1573-81

16. DeLoach LJ, Higgins MS, Caplan AB, Stiff JL. The visual analog scale in the immediate postoperative period: intrasubject variability and correlation with a numeric scale. Anesth Analg. 1998; 86: 102-6

17. Dexter F, Chestnut DH. Analysis of statistical tests to compare visual analog scale measurements among groups. Anesthesiology. 1995; 82: 896-902
18. Myles PS, Troedel S, Boquest M, Reeves M. The pain visual analog scale: is it linear or nonlinear? Anesth Analg. 1999; 89: 1517-20

19. Robaina F. ¿Por qué las Unidades del Dolor deben ser multidisciplinarias? Rev. Soc. Esp. Dolor. 2005; 12: 137-140

20. Allegri M, Clark MR, De Andrés J, Jensen TS. Acute and chronic pain: where we are and where we have to go. Minerva Anestesiol. 2012; 78(2): 222-35

21. Cardona E, Castaño M, Builes A, Castro G. Manejo del dolor postquirúrgico en el Hospital Universitario San Vicente de Paul de Medellín. Rev. Col. Anest. 2003; 31: 111-6

22. López S, Méndez H, Real J, Gordo F, Fernández DL, Manejo de la analgesia postoperatoria en las primeras 24 horas en un Hospital de segundo nivel. Rev. Soc. Esp. Dolor. 2006; 13: 18-23

23. Gallego JI, Rodriguez de la Torre MR, Vazquez-Guerrero JC, Gil M. Estimation of the prevalence and severity of postoperative pain and relation with patient satisfaction. Rev. Soc. Esp. Dolor. 2004; 11:197-202

24. Cadavid A, Mendoza J, Gómez N, Berrío M. Prevalencia de dolor agudo posoperatorio y calidad de la recuperación en el Hospital Universitario San Vicente de Paúl, Medellín, Colombia, 2007. IATREIA. 2009; 22: 11-5

25. Rocchi A, Chung F, Forte L. Canadian survey of postsurgical pain and pain medication experiences. Can J Anaesth. 2002; 49(10): 1053-6.

26. Manion SC, Brennan TJ. Thoracic epidural analgesia and acute pain management. Anesthesiology. 2011; 115(1): 181-8

27. Moreno-Azcoitia M, De Andrés J, Torres L, Vidal M. Estudio observacional sobre el dolor postoperatorio leve o moderado desde el punto de vista del anestesiólogo en España. PATHOS. Rev. Soc. Esp. Dolor. 2007; 14: 550-67

28. Morrison RS, Magaziner J, Gilbert M, Koval KJ. McLaughlin MA, Orosz G, et al. Relationship between pain and opioid analgesics on the development of delirium following hip fracture. J Gerontol A Biol Sci Med Sci. 2003; 58 (1): 76-81.

29. Valentín López B, García Caballero J, Muñoz Ramón JM, Aparicio Grande P, Díez Sebastán J, Criado Jiménez A. [Postoperative pain management in a tertiary care hospital: initial situation prior to starting a quality assurance program]. Rev Esp Anestesiol Reanim. 2006; 53(7): 408-18.

30. Brennan F, Cousins MJ. Pain relief as a human right. Rev. Soc. Esp. Dolor. 2005; 12: 17-23.

31. Soler E, Faus MT, Montaner MC, Morales F, Martínez-Pons V. Prevalencia, tratamiento y factores determinantes del dolor postoperatorio en un servicio de cirugía general y aparato digestivo. Rev. Soc. Esp. Dolor. 2001; 8 (5): 317-26.

32. Machado-Alba J, Quintero A, Mena M, Castaño C, Lopez E, Marin D, et al. Evaluación del manejo del dolor postquirúrgico en pacientes adultos de una clínica de tercer nivel de Pereira, Colombia. Rev Investigaciones Andina. 2012; 14: 547-59. 